\title{
On angular localization of spectra of perturbed operators
}

\author{
M.I. GIL' \\ Department of Mathematics, Ben Gurion University of the Negev \\ P.O. Box 653, Beer-Sheva 84105, Israel
}

gilmi@bezeqint.net

Received June 29, 2020

Accepted September 17, 2020

Presented by Manuel González

Abstract: Let $A$ and $\tilde{A}$ be bounded operators in a Hilbert space. We consider the following problem: let the spectrum of $A$ lie in some angular sector. In what sector the spectrum of $\tilde{A}$ lies if $A$ and $\tilde{A}$ are "close"? Applications of the obtained results to integral operators are also discussed.

Key words: Operators, spectrum, angular location, perturbations, integral operator.

AMS Subject Class. (2010): 47A10, 47A55, 47B10.

\section{INTRODUCTION AND PRELIMINARIES}

Let $\mathcal{H}$ be a complex separable Hilbert space with a scalar product $(.,$.$) ,$ the norm $\|\|=.\sqrt{(., .)}$ and unit operator $I$. By $\mathcal{B}(\mathcal{H})$ we denote the set of bounded operators in $\mathcal{H}$. For an $A \in \mathcal{B}(\mathcal{H}), A^{*}$ is the adjoint operator, $\|A\|$ is the operator norm and $\sigma(A)$ is the spectrum.

We consider the following problem: let $A$ and $\tilde{A}$ be "close" operators and $\sigma(A)$ lie in some angular sector. In what sector $\sigma(\tilde{A})$ lies?

Not too much works are devoted to the angular localizations of spectra. The papers [5, 6, 7, 8] should be mentioned. In particular, in the papers by E.I. Jury, N.K. Bose and B.D.O. Anderson [5, 6] it is shown that the test to determine whether all eigenvalues of a complex matrix of order $n$ lie in a certain sector can be replaced by an equivalent test to find whether all eigenvalues of a real matrix of order $4 n$ lie in the left half plane. The results from [5] have been applied by G.H. Hostetter [4] to obtain an improved test for the zeros of a polynomial in a sector. In [7] M.G. Krein announces two theorems concerning the angular localization of the spectrum of a multiplicative operator integral. In the paper 8 G.V. Rozenblyum studies the asymptotic behavior of the distribution functions of eigenvalues that appear in a fixed angular region of the complex plane for operators that are close to normal. As applications, he calculates the asymptotic behavior of the spectrum of two classes of oper- 
ators: elliptic pseudo-differential operators acting on the sections of a vector bundle over a manifold with a boundary, and operators of elliptic boundary value problems for pseudo-differential operators. It should be noted that in the just pointed papers the perturbations of an operator whose spectrum lie in a given sector are not considered. Below we give bounds for the spectral sector of a perturbed operator.

Without loss of the generality it is assumed that

$$
\beta(A):=\inf \operatorname{Re} \sigma(A)>0 .
$$

If this condition does not hold, instead of $A$ we can consider perturbations of the operator $A_{1}=A+I c$ with a constant $c>|\beta(A)|$.

For a $Y \in \mathcal{B}(\mathcal{H})$ we write $Y>0$ if $Y$ is positive definite, i.e., $\inf _{x \in \mathcal{H},\|x\|=1}$ $(Y x, x)>0$. Let $Y>0$. Define the angular $Y$-characteristic $\tau(A, Y)$ of $A$ by

$$
\cos \tau(A, Y):=\inf _{x \in \mathcal{H},\|x\|=1} \frac{\operatorname{Re}(Y A x, x)}{|(Y A x, x)|} .
$$

The set

$$
S(A, Y):=\{z \in \mathbb{C}:|\arg z| \leq \tau(A, Y)\}
$$

will be called the $Y$-spectral sector of $A$.

Lemma 1.1. For an $A \in \mathcal{B}(\mathcal{H})$, let condition (1.1) hold and $Y$ be a positive definite operator, such that $(Y A)^{*}+Y A>0$. Then $\sigma(A)$ lies in the $Y$-spectral sector of $A$.

Proof. Take a ray $z=r e^{i t}(0<r<\infty)$ intersecting $\sigma(A)$, and take the point $z_{0}=r_{0} e^{i t}$ on it with the maximum modulus. By the theorem on the boundary point of the spectrum [1, Section I.4.3, p. 28] there exists a normed sequence $\left\{x_{n}\right\}$, such that $A x_{n}-z_{0} x_{n} \rightarrow 0,(n \rightarrow \infty)$. Hence,

$$
\frac{\operatorname{Re}\left(Y A x_{n}, x_{n}\right)}{\left|\left(Y A x_{n}, x_{n}\right)\right|}=\frac{\operatorname{Re} r_{0} e^{i t}\left(Y x_{n}, x_{n}\right)}{r_{0}\left|\left(Y x_{n}, x_{n}\right)\right|}+\epsilon_{n}=\cos t+\epsilon_{n}
$$

with $\epsilon_{n} \rightarrow 0$ as $n \rightarrow \infty$. So $z_{0}$ is in $S(A, Y)$. This proves the lemma.

Example 1.2. Let $A=A^{*}>0$. Then condition (1.1) holds. For any $Y>0$ commuting with $A$ (for example $Y=I$ ) we have $(Y A)^{*}+Y A=2 Y A$ and $\operatorname{Re}(Y A x, x)=|(Y A x, x)|$. Thus $\cos \tau(A, Y)=1$ and $S(A, Y)=\{z \in \mathbb{C}:$ $\arg z=0\}$. 
So Lemma 1.1 is sharp.

Remark 1.3. Suppose $A$ has a bounded inverse. Recall that the quantity $\operatorname{dev}(A)$ defined by

$$
\cos \operatorname{dev}(A):=\inf _{x \in \mathcal{H}, x \neq 0} \frac{\operatorname{Re}(A x, x)}{\|A x\|\|x\|}
$$

is called the angular deviation of $A$, cf. [1, Chapter 1, Exercise 32]. For example, for a positive definite operator $A$ one has

$$
\cos \operatorname{dev}(A)=\frac{2 \sqrt{\lambda_{M} \lambda_{m}}}{\lambda_{M}+\lambda_{m}}
$$

where $\lambda_{m}$ and $\lambda_{M}$ are the minimum and maximum of the spectrum of $A$, respectively (see [1, Chapter 1, Exercise 33]). Besides, in Exercise 32 it is pointed that the spectrum of $A$ lies in the sector $|\arg z| \leq \operatorname{dev}(A)$. Since $|(A x, x)| \leq\|A x\|\|x\|$, Lemma 1.1 refines the just pointed assertion.

\section{The MAIn Result}

Let $A$ be a bounded linear operator in $\mathcal{H}$, whose spectrum lies in the open right half-plane. Then by the Lyapunov theorem, cf. [1, Theorem I.5.1], there exists a positive definite operator $X \in \mathcal{B}(\mathcal{H})$ solving the Lyapunov equation

$$
2 \operatorname{Re}(A X)=X A+A^{*} X=2 I .
$$

So $\operatorname{Re}(X A x, x)=\left(\left(X A+A^{*} X\right) x, x\right) / 2=(x, x)(x \in \mathcal{H})$ and

$$
\cos \tau(A, X)=\inf _{x \in \mathcal{H},\|x\|=1} \frac{(x, x)}{|(X A x, x)|}=\frac{1}{\sup _{x \in \mathcal{H},\|x\|=1}|(X A x, x)|} \geq \frac{1}{\|A X\|} .
$$

Put

$$
J(A)=2 \int_{0}^{\infty}\left\|e^{-A t}\right\|^{2} d t .
$$

Now we are in a position to formulate our main result.

Theorem 2.1. Let $A, \tilde{A} \in \mathcal{B}(\mathcal{H})$, condition (1.1) hold and $X$ be a solution of (2.1). Then with the notation $q=\|A-\tilde{A}\|$ one has

$$
\cos \tau(\tilde{A}, X) \geq \cos \tau(A, X) \frac{(1-q J(A))}{(1+q J(A))},
$$

provided

$$
q J(A)<1 .
$$


The proof of this theorem is based on the following lemma.

Lemma 2.2. Let $A, \tilde{A} \in \mathcal{B}(\mathcal{H})$, condition (1.1) hold and $X$ be a solution of (2.1). If, in addition,

$$
q\|X\|<1,
$$

then

$$
\cos \tau(\tilde{A}, X) \geq \cos \tau(A, X) \frac{(1-\|X\| q)}{(1+\|X\| q)}
$$

Proof. Put $E=\tilde{A}-A$. Then $q=\|E\|$ and due to (2.1), with $x \in \mathcal{H}$, $\|x\|=1$, we obtain

$$
\begin{aligned}
\operatorname{Re}(X(A+E) x, x) & \geq \operatorname{Re}(X A x, x)-|(X E x, x)| \\
& =(x, x)-|(X E x, x)| \\
& \geq(x, x)-\|X\|\|E\|\|x\|^{2}=1-\|X\| q .
\end{aligned}
$$

In addition,

$$
\begin{aligned}
|(X(A+E) x, x)| & \leq|(X A x, x)|+\|X\|\|E\|\|x\|^{2} \\
& =|(X A x, x)|\left(1+\frac{\|X\| q}{|(X A x, x)|}\right) \quad(\|x\|=1) .
\end{aligned}
$$

But

$$
|(X A x, x)| \geq|\operatorname{Re}(X A x, x)|=\operatorname{Re}(X A x, x)=(x, x)=1 .
$$

Hence

$$
|(X(A+E) x, x)| \leq|(X A x, x)|\left(1+\frac{\|X\| q}{\operatorname{Re}(X A x, x)}\right) \leq|(X A x, x)|(1+\|X\| q) .
$$

Now (2.3) yields.

$$
\frac{\operatorname{Re}(X \tilde{A} x, x)}{|(X \tilde{A} x, x)|} \geq \frac{(1-\|X\| q)}{|(X A x, x)|(1+\|X\| q)} \quad(\|x\|=1),
$$

provided $(2.2)$ holds. Since

$$
\cos \tau(\tilde{A}, X)=\inf _{x \in \mathcal{B},\|x\|=1} \frac{\operatorname{Re}(X \tilde{A} x, x)}{|(X \tilde{A} x, x)|}
$$

we arrive at the required result. 
Proof of Theorem 2.1 Note that $X$ is representable as

$$
X=2 \int_{0}^{\infty} e^{-A^{*} t} e^{-A t} d t
$$

[1, Section 1.5]. Hence, we easily have $\|X\| \leq J(A)$. Now the latter lemma proves the theorem.

\section{Operators with Hilbert-Schmidt Hermitian components}

In this section we obtain an estimate for $J(A)(A \in \mathcal{B}(\mathcal{H}))$ assuming that $A \in \mathcal{B}(\mathcal{H})$ and

$$
A_{I}:=\left(A-A^{*}\right) / i \quad \text { is a Hilbert-Schmidt operator, }
$$

i.e., $N_{2}\left(A_{I}\right):=\left(\operatorname{trace}\left(A_{I}^{2}\right)\right)^{1 / 2}<\infty$. Numerous integral operators satisfy this condition. Introduce the quantity (the departure from normality)

$$
g_{I}(A):=\left[2 N_{2}^{2}\left(A_{I}\right)-2 \sum_{k=1}^{\infty}\left|\operatorname{Im} \lambda_{k}(A)\right|^{2}\right]^{1 / 2} \leq \sqrt{2} N_{2}\left(A_{I}\right),
$$

where $\lambda_{k}(A)(k=1,2, \ldots)$ are the eigenvalues of $A$ taken with their multiplicities and ordered as $\left|\operatorname{Im} \lambda_{k+1}(A)\right| \leq\left|\operatorname{Im} \lambda_{k}(A)\right|$. If $A$ is normal, then $g_{I}(A)=0$, cf. [2, Lemma 9.3].

Lemma 3.1. Let conditions (1.1) and (3.1) hold. Then $J(A) \leq \hat{J}(A)$, where

$$
\hat{J}(A):=\sum_{j, k=0}^{\infty} \frac{g_{I}^{j+k}(A)(k+j) !}{2^{j+k} \beta^{j+k+1}(A)(j ! k !)^{3 / 2}} .
$$

Proof. By [2, Theorem 10.1] we have

$$
\left\|e^{-A t}\right\| \leq \exp [-\beta(A) t] \sum_{k=0}^{\infty} \frac{g_{I}^{k}(A) t^{k}}{(k !)^{3 / 2}} \quad(t \geq 0) .
$$


Then

$$
\begin{aligned}
J(A) & \leq 2 \int_{0}^{\infty} \exp [-2 \beta(A) t]\left(\sum_{k=0}^{\infty} \frac{g_{I}^{k}(A) t^{k}}{(k !)^{3 / 2}}\right)^{2} d t \\
& =2 \int_{0}^{\infty} \exp [-2 \beta(A) t]\left(\sum_{j, k=0}^{\infty} \frac{g_{I}^{k+j}(A) t^{k+j}}{(j ! k !)^{3 / 2}}\right) d t \\
& =\sum_{j, k=0}^{\infty} \frac{2(k+j) ! g_{I}^{j+k}(A)}{(2 \beta(A))^{j+k+1}(j ! k !)^{3 / 2}},
\end{aligned}
$$

as claimed.

If $A$ is normal, then $g_{I}(A)=0$ and with $0^{0}=1$ we have $\hat{J}(A)=\frac{1}{\beta(A)}$.

The latter lemma and Theorem 2.1 imply

Corollary 3.2. Let $A, \tilde{A} \in \mathcal{B}(\mathcal{H})$ and let the conditions (1.1), (3.1) and $q \hat{J}(A)<1$ hold. Then

$$
\cos \tau(\tilde{A}, X) \geq \frac{(1-q \hat{J}(A))}{(1+q \hat{J}(A))} \cos \tau(A, X) .
$$

\section{INTEGRAL OPERATORS}

As usually $L^{2}=L^{2}(0,1)$ is the space of scalar-valued functions $h$ defined on $[0,1]$ and equipped with the norm

$$
\|h\|=\left[\int_{0}^{1}|h(x)|^{2} d x\right]^{1 / 2} .
$$

Consider in $L^{2}(0,1)$ the operator $\tilde{A}$ defined by

$$
(\tilde{A} h)(x)=a(x) h(x)+\int_{0}^{1} k(x, s) h(s) d s \quad\left(h \in L^{2}, x \in[0,1]\right),
$$

where $a(x)$ is a real bounded measurable function with

$$
a_{0}:=\inf a(x)>0,
$$

and $k(x, s)$ is a scalar kernel defined on $0 \leq x, s \leq 1$, and

$$
\int_{0}^{1} \int_{0}^{1}|k(x, s)|^{2} d s d x<\infty .
$$


So the Volterra operator $V$ defined by

$$
(V h)(x)=\int_{x}^{1} k(x, s) h(s) d s \quad\left(h \in L^{2}, x \in[0,1]\right),
$$

is a Hilbert-Schmidt one. Define operator $A$ by

$$
(A h)(x)=a(x) h(x)+\int_{x}^{1} k(x, s) h(s) d s \quad\left(h \in L^{2}, x \in[0,1]\right) .
$$

Then $A=D+V$, where $D$ is defined by $(D h)(x)=a(x) h(x)$. Due to Lemma 7.1 and Corollary 3.5 from [3] we have $\sigma(A)=\sigma(D)$. So $\sigma(A)$ is real and $\beta(A)=a_{0}$. Moreover,

$$
N_{2}\left(A_{I}\right)=N_{2}\left(V_{I}\right) \leq N_{2}(V)=\left[\int_{0}^{1} \int_{x}^{1}|k(x, s)|^{2} d s d x\right]^{1 / 2} .
$$

Here $V_{I}=\left(V-V^{*}\right) / 2 i$. Thus,

$$
g_{I}(A) \leq g_{V}:=\sqrt{2} N_{2}(V)
$$

and

$$
\|A-\tilde{A}\| \leq q_{0}:=\left[\int_{0}^{1} \int_{0}^{x}|k(x, s)|^{2} d s d x\right]^{1 / 2} .
$$

Simple calculations show that under consideration

$$
\hat{J}(A) \leq \hat{J}_{0}:=\sum_{j, k=0}^{\infty} \frac{g_{V}^{j+k}(k+j) !}{2^{j+k} a_{0}^{j+k+1}(j ! k !)^{3 / 2}} .
$$

Making use of Corollary 3.2 and taking into account that in the considered case $\cos \tau(A, X)=1$, we arrive at the following result.

Corollary 4.1. Let $\tilde{A}$ be defined by (4.1) and the conditions (4.2) and (4.3) hold. If, in addition, $q_{0} \hat{J}_{0}<1$, then $\sigma(A)$ lies in the angular sector

$$
\left\{z \in \mathbb{C}:|\arg z| \leq \arccos \frac{\left(1-q_{0} \hat{J}_{0}\right)}{\left(1+q_{0} \hat{J}_{0}\right)}\right\} .
$$

EXAmPLE 4.2. To estimate the sharpness of our results consider in $L^{2}(0,1)$ the operators

$$
(A h)(x)=2 h(x) \text { and }(\tilde{A} h)(x)=(2+i) h(x) \quad\left(h \in L^{2}, x \in[0,1]\right) .
$$


$\sigma(A)$ consists of the unique point $\lambda=2$ and so $\cos (A, X)=\cos \arg \lambda=1$. We have

$$
J(A)=2 \int_{0}^{\infty} e^{-4 t} d t=1 / 2 \quad \text { and } \quad q=1 .
$$

By Corollary 3.2

$$
\cos \tau(\tilde{A}, X) \geq \frac{1-1 / 2}{1+1 / 2}=1 / 3 .
$$

Compare this inequality with the sharp result: $\sigma(\tilde{A})$ consists of the unique point $\tilde{\lambda}=2+i$. So $\tan (\arg \tilde{\lambda})=1 / 2$, and therefore $\cos (\arg \tilde{\lambda})=2 /(\sqrt{5})$.

\section{ACKNOWLEDGEMENTS}

I am very grateful to the referee of this paper for his (her) deep and helpful remarks.

\section{REFERENCES}

[1] Yu.L. Daleckit, M.G. Krein, "Stability of Solutions of Differential Equations in Banach Space", Vol. 43, American Mathematical Society, Providence, R. I., 1974.

[2] M.I. GIL', "Operator Functions and Operator Equations", World Scientific Publishing Co. Pte. Ltd., Hackensack, New Jersey, 2018.

[3] M.I. GIL', Norm estimates for resolvents of linear operators in a Banach space and spectral variations, Adv. Oper. Theory 4(1) (2019), 113-139.

[4] G.H. Hostetter, An improved test for the zeros of a polynomial in a sector, IEEE Trans. Automatic Control AC-20 (3) (1975), 433-434.

[5] E.I. Jury, N.K. Bose, B.D.O. Anderson, A simple test for zeros of a complex polynomial in a sector, IEEE Trans. Automatic Control AC-19 (1974), 437-438.

[6] E.I. Jury, N.K. Bose, B.D.O. Anderson, On eigenvalues of complex matrices in a sector, IEEE Trans. Automatic Control AC-20 (1975), 433434.

[7] M.G. Krein, The angular localization of the spectrum of a multiplicative integral in Hilbert space (in Russian) Funkcional. Anal. i Prilozhen $\mathbf{3}(1)$ (1969), 89-90.

[8] G.V. Rozenblyum, Angular asymptotics of the spectrum of operators that are close to normal, J. Soviet Math. 45 (3) (1989), 1250-1261. 